\title{
Hacia una sociedad ambiental
}

\author{
Elisabet Tejero Gil \\ Universitat Autònoma de Barcelona. Departament de Sociologia \\ 08193 Bellaterra (Barcelona). Spain
}

\section{Resumen}

El deterioro progresivo del medio ambiente y de la calidad de vida de las mayorías sigue obligando a hacer una reflexión profunda sobre la acción que la sociedad ejerce sobre el entorno en el que vive. El modelo de desarrollo hoy vigente en los países industrializados y en los países dependientes está en el punto de mira de esta reflexión.

Con este artículo se participa en el debate actual aportando algunos elementos teóricometodológicos para contribuir a la formación de una perspectiva de análisis medioambiental capaz de abordar el estudio de los procesos sociales, tanto a escala local como a escala global o planetaria.

El concepto de medio ambiente está estrechamente ligado al concepto y al hecho social de habitar, actividad básica y compleja de la humanidad. De ahí que el título del artículo, "Hacia una sociedad ambiental", sea más que un ejercicio teórico, una apuesta reflexiva sobre el desarrollo.

Palabras clave: medio ambiente, ecología, desarrollo, habitar, identidad y mente local.

\section{Abstract: Towards an environment society}

Today society is forced to reflect on the increasing of environment damage and its impact on welfare. The present development pattern in both, industrialized and dependent countries, is the main focus of this discussion.

The article tries to make a contribution to the present debate, by introducing some theotetical and methodological elements, thus enabling to create an environmental perspective for analysing social processes in a local and global scale.

The concept of environment is closely linked to the concept and social process of dwelling, which is a basic and complex activity of human beings. Therefore the tittle of this article, that is "Towards an envitonment society", tries to show the conflictive relationship between this activity and development processes.

Key words: environment, ecology, development, dwelling, identity and local mind.

\section{Sumari}

\section{Introducción}

2. ¿Qué entendemos cuando hablamos de medio ambiente

3. ¿Qué es una perspectiva de análisis medioambiental?
4. Una propuesta metodológica para un paradigma medioambiental Bibliografía 


\section{Introducción}

Los movimientos conservacionistas iniciaron desde el siglo pasado una reflexión sobre las consecuencias, muchas veces irreversibles, de la actividad humana sobre los sistemas naturales. Actualmente el deterioro progresivo del medio ambiente y de la calidad de vida de las mayorías sigue obligando a hacer una reflexión profunda sobre este impacto perjudicial para nuestra supervivencia.

Cada vez más los movimientos ambientales han ido comprendiendo que el deterioro ambiental está íntimamente ligado al estilo de desarrollo, tanto de los países industrializados como de los países dependientes. Desde este punto de vista, la problemática ambiental se considera como el resultado de la actividad humana sobre el ecosistema. Dicha actividad, que se basa en el avance tecnológico, está orientada por los criterios y los objetivos que rigen el desarrollo en cada formación social.

Desde diferentes disciplinas nos vemos abocados a un nuevo paradigma comprensivo, denominado paradigma medioambientalista. Por un lado, recoge el análisis y la crítica del tipo de desarrollo en el cual está inmersa la humanidad. Por otro, las propuestas hacia un tipo de desarrollo equilibrado y sostenible entre la sociedad y los recursos naturales que necesita. Este paradigma nos empuja a salir de esos compartimentos estancos que son las especialidades profesionales y científicas. Sin dejar atrás esa identidad que nos afanamos tanto en preservar, esta perspectiva impone como método la interdisciplinariedad. De ella se pretende que surja una visión dinámica, integral e integrada de los modos de vida actuales, de sus antecedentes y de su capacidad de proyectarse en el futuro.

La ventaja que presenta esta perspectiva de análisis es que está en plena fase de formación y eso nos permite poder participar en el proceso de su construcción. Pero además su propia constitución interna, al integrar diferentes disciplinas y apostar por visiones dinámicas de la realidad, permite situarse frente a una perspectiva de análisis y de intervención abierta y en continuo proceso de formación. Así pues, este artículo está pensado como una aventura. La aventura de participar en la creación y en la recreación de esta perspectiva aportando elementos conceptuales y metodológicos que pueden ayudar a situar y esclarecer de qué hablamos cuando nos referimos a un paradigma o a una perspectiva medioambiental.

\section{2. ¿Qué entendemos cuando hablamos de medio ambiente?}

Tanto en los medios institucionales y científicos como en los populares, la referencia al medio ambiente se identifica en primer término con los recursos naturales que necesitamos para nuestra vida y que utilizamos cotidianamente: el agua, la luz solar, la tierra, la vegetación. Esta concepción corre el riesgo, en ocasiones, de crear la imagen de un medio ambiente «naturalista» que concibe a la persona al margen, distanciada de la naturaleza. El mito de la tecnología, la visión de un progreso imparable, incuestionado y salvador, ha lle- 
vado a que se asuman posturas prepotentes respecto a la utilización de los recursos de nuestro entorno. Se vive de la fantasía de someter a la naturaleza a nuestros designios porque nosostros estamos "aquí" y ella está "ahí" para servirnos.

Este separarse e independizarse del entorno ha sido una de las principales consecuencias que han tenido los procesos de industrialización de este siglo, basados en criterios de homogeneidad y estandarización de las relaciones socioespaciales. La perspectiva medioambiental rechaza esta visión enajenada de la persona respecto a la naturaleza. El ser humano es una parte de esta naturaleza, si bien una parte fundamental por su capacidad de manipulación. El ser humano está en permanente relación de intercambio y transformación recíproca con la naturaleza. Así pues, el medio ambiente no es sólo la naturaleza, es el medio o espacio antropizado, es decir, todo lugar afectado por la intervención humana. Este concepto abarca tanto los lugares habitados como los espacios que directa o indirectamente están recibiendo la influencia de cualquier actividad humana. Este enfoque considera que toda actividad humana es la manifestación de un modo de vida y éste, a su vez, expresión de la cultura de una sociedad. Hay que recalcar que se trata de la sociedad de un determinado lugar, es decir, identificada por unas características sociales, económicas, políticas, históricas y físicas propias.

Así pues, queda claro que medio ambiente no es el campo en oposición a la urbe sino que ambas son medio ambiente con sus condiciones y condicionantes específicos. Incluso, como objetos de análisis, el campo o la ciudad mueren en sí mismos si no se rebasa su frontera física para dar paso a un análisis de los modos de vida que se da en cada uno, y cómo se influyen mutuamente. Actualmente, cuando las ciudades están absorbiendo la mayoría de la población del planeta, la piedra de toque está en los procesos de urbanización, que son expresión de las formas de vida urbanas.

Medio ambiente es el resultado de la relación del individuo, la comunidad y la sociedad con el entorno físico donde se asientan y donde habitan. Esta relación se estructura en función de la cultura que fundamenta la utilización material de los recursos y la composición simbólica de los mecanismos de apropiación de los mismos.

\section{3. ¿Qué es una perspectiva de análisis medioambiental?}

Las formas en las que se han venido entendiendo las relaciones sociales en los diferentes ámbitos territoriales, urbanos o rurales, han llegado a un punto crítico, sobre todo en la esfera de la planificación. Los análisis más puramente funcionalistas se han quedado en un conocimiento superficial de la relación de los individuos con su entorno, y en los tiempos del movimiento moderno han acuñado una forma de asentamientos humanos tendentes a la homogeneización y a la pérdida de memoria e identidad.

Reflexionar desde una perspectiva medioambiental implica buscar un marco de análisis e interpretación de las relaciones sociales y su vinculación al entorno y al tipo de desarrollo que en él se realiza. Se hace necesario superar cier- 
tas limitaciones de perspectivas materialistas que han explicado los procesos sociales ligados al territorio con una óptica marcadamente economicista, y de perspectivas subjetivistas decantadas hacia una visión eminentemente culturalista del territorio.

Un marco de referencia útil que permite indagar sobre el tipo de pensamiento que estructura el paradigma medioambiental, es la distinción que Pascal hace de dos tipos de mentes: la mente amplia y la mente geométrica (F. La Cecla, 1993). La mente amplia es capaz de comprender un gran número de principios sin confundirlos. Se trata de una mente que se construye a partir de la historia y que se estructura en función de imágenes visibles, palpables, tangibles y llenas de vida. Son imágenes claras y detalladas de una escena donde hay multiplicidad de agentes en acción. La mente geométrica es capaz de penetrar rápida e incisivarnente en las consecuencias de los principios. Se basa en nociones abstractas y se estructura en función de categorías como el orden o la simplicidad. Mientras que la primera no tiene paciencia de descender a los principios primeros de los objetos especulativos y que están fuera del modo de ser ordinario de las cosas, la segunda se retrae cuando se encuentra con una multiplicidad de proposiciones cuya diversidad y complejidad no entiende.

Ante ambas, la perspectiva medioambientalista adopta fundamentalmente el tipo de mente amplia, que es capaz de abrirse a multitud de visiones y que compone la realidad a partir del principio de complejidad. Según este tipo de pensamiento, los fenómenos sociales como el habitar o el ambientarse se entienden y se explican no sólo con el intelecto sino también con dos elementos más: las emociones y la imaginación. Sólo con las categorías analíticas y racionales no es posible entender globalmente un lugar, las formas de vida que en él se dan. Para captarlo en su globalidad se tiene que recurrir además a las dimensiones simbólicas, afectivas, a las ilusiones y esperanzas que se proyectan en él.

La relación con el medio ambiente es una dimensión vivida de nuestra existencia y, como tal, primero la percibirnos, la sentimos y después la racionalizamos. Relegar a cualquiera de estos elementos de la comprensión del medio ambiente significa perder la globalidad del fenómeno y también los matices y las especificidades que lo caracterizan y lo individualizan. La perspectiva medioambiental recoge entre sus guías el esquema conceptual de la ecología. Esta es la parte de la biología que estudia las relaciones existentes entre los organismos y el medio en el que viven. Adaptada a las ciencias sociales, la ecología estudia las relaciones entre los seres humanos y el medio donde desarrollan su vida.

De la ecología se adaptan los siguientes criterios y conceptos:

a) Una visión dinámica de las relaciones entre seres humanos y medio, siempre en continuo proceso de adaptación, transformación o cambio.

b) La idea de sistema expresada por el concepto de ecosistema, es decir el medio o "casa" donde se desarrollan los organismos vivos. Todos los ele- 
mentos del mismo están interrelacionados y cualquier alteración de uno se traduce en una alteración para el conjunto.

c) La idea de equilibrio, ya que las relaciones en los ecosistemas tienden al equilibrio o a la armonía dinámica.

d) El concepto de umbral de sensibilidad, que marca el límite de resistencia entre un proceso de transformación continua en la relación de los individuos con el medio y un proceso de cambio hacia otra forma de estructurar estas relaciones.

Además de estos elementos que aporta la ecología, un análisis medioambiental parte de cuatro conceptos marco:

a) El criterio de globalidad: como primer paso para comprender la estructura y la dinámica del medio ambiente, acotado al ecosistema que se estudie, es necesario tener una visión del conjunto y aprehender su complejidad, ya que la realidad no se concibe como una agregación de partes sino como un todo.

b) El criterio de interdependencia: todos los elementos del ecosistema están relacionados unos con otros y en mayor o menor medida dependen entre ellos para su devenir. No hay pues entidades aisladas y absolutamente autónomas, ya que se requiere del flujo de otras entidades para la subsistencia.

c) El criterio de reciprocidad: las relaciones entre los individuos y su hábitat están en continuo intercambio y todo aquello que se recibe se retorna transformado. A esta propiedad se suma la de la multiplicidad de influencias o inputs que los individuos y el hábitat reciben y, por lo tanto, la multiplicidad de efectos que cada uno de ellos desprende.

Puede parecer que lo expuesto hasta el momento supera en poco a la conocida ecología humana, que surge de la Escuela de Chicago y tiene valiosos trabajos que explican los lugares a través de las funciones alli desarrolladas o a través de las percepciones y sensaciones crecidas al amparo de un determinado espacio (urbano). Estos trabajos tienen como telón de fondo una concepción funcionalista de las relaciones socioespaciales. Sin embargo, la perspectiva medioambiental, en su búsqueda de una explicación y de una interpretación de los diferentes modos de desarrollo, utiliza como soporte la teoría de la producción social del espacio desarrollada por $\mathrm{H}$. Lefebvre y M. Castells, pero incluye una nueva cuña: la del proceso cultural de construcción de los imaginarios del lugar, del espacio vivido (A. Silva, 1992).

La perspectiva medioambiental requiere de una teoría o marco conceptual de la relación entre el espacio y la sociedad que le permita entender las relaciones constitutivas de una formación social dada en un espacio determinado. Así pues, se trata de un abordaje al espacio como categoría social. "La organización del espacio es una manifestación de la transformación de la naturaleza 
por la sociedad humana” (M. Acosta, 1987). Así pues, objetos de estudio fundamentales para una concepción medjoambiental son:

- cómo una sociedad se produce espacialmente;

- cómo ese espacio es expresión, sustento y lugar de lucha por la reproducción de esa sociedad;

- qué tipo de desarrollo social implica.

El estudio de una comunidad, de una sociedad, es indisociable del espacio que la sustenta. De su relación dialéctica surge el proceso de apropiación del espacio que significa (R. Briceño, 1987):

- Conocer un espacio con los recursos materiales y simbólicos que ofrece y reconocerlo como parte inherente a las formas de vida específicas que en él se desarrollan;

- Hacerlo propio ocupándolo y participando de su dinámica al utilizar los recursos que brinda para la subsistencia material y subjetiva (valores y emociones);

- Devolver al espacio el producto de la intervención humana en el mismo.

La apropiación del espacio es un fenómeno cultural en el cual un individuo, un grupo, una comunidad o una sociedad construye las directrices de cuáles son los recursos apropiables (imputs), cómo apropiarlos (proceso) y el tipo de transformaciones asumibles (outputs), tanto por la sociedad como por los espacios donde se interviene.

La simbiosis entre espacio (recursos) y sociedad (cultura, en su acepción más amplia)) se concreta en el concepto de habitar o morar y ésta es la matriz que ampara los análisis medioambientales. Habitar no es un fenómeno que se pueda definir diciendo, simplemente, que es el hecho de estar en un lugar, así como tampoco sirve definir el hábitat como un asentamiento humano. Desde una concepción más amplia, y también más abstracta pero con más posibilidades de comprender el fenómeno en su globalidad y diversidad, se puede decir que habitar es darse cuenta del aquí.

Es un proceso cognitivo a través del cual se hace evidente el espacio y pasa de ser una categoría que describe "el continente», "el significante», para dar a luz información sobre "el contenido", "el significado". De espacio se pasa a lugar. De una dimensión abstracta se pasa a una vivida. Así pues, habitar es una facultad humana que no es ni dada ni inmediata. Este proceso de conocimiento desarrollado por el que habita es el proceso de apropiación del espacio. Habitar es "vivir de un lugar", hacerlo propio para asegurar la subsistencia no sólo económica sino también cultural.

Habitar implica el nacimiento de una presencia reconocida en un lugar y el instaurar relaciones de familiariedad con el entorno, de intimidad en ese conocimiento mutuo y en esa interdependencia de usos para sobrevivir, de interioridad y de recogimiento. Esto sería lo que se expresa popularmente cuando decimos "hacerse con el lugar". La consciencia del habitar, es decir, el paso de la visión abstracta del espacio a la concreción de las condiciones de vida en 
el lugar, se construye individual y colectivamente a través de lo que se ha denominado la mente local.

Desarrollar la mente local es depositar la mente propia en un lugar. Este tipo de mente está ligado a lo local, al aquí. El que esta categoría tenga entidad por sí misma y no se desvanezca de generación en generación tiene sus raíces en la "memoria» que se transmite de forma casi imperceptible como una herencia del lugar. Utilizar unos recursos y no otros, darles unos usos determinados y estructurar en función de ello la vida cotidiana y el sentido de su devenir, construyen una mente local. A la vez, este proceso se convierte en una plataforma para la construcción de una identidad en tanto es un proceso para identificarse con el lugar y desarrollar un sentido de la "pertenencia a».

Dentro de esta perspectiva medioambiental el concepto de mente local es primordial para entender que el habitar es un proceso que responde a la diversidad cultural y, más concretamente, a las especificidades locales. Otra cuestión es que el tratamiento dado este último siglo a la técnica y a la industrialización ha conducido a una concepción del espacio como categoría neutra. El coste de esta concepción ha sido la construcción de espacios inapropiables por sus pobladores y el olvido de la mente local. La construcción de la mente local no es un proceso definido por la exclusión hacia otras mentes locales. La identidad de un lugar no está basada en la contraposición respecto a la identidad de otro lugar. La exacerbación de lo local, la desigualdad basada en la identidad, son fenómenos que hoy se viven y que van más allá de la mente local. Éstos se sitúan en la esfera de la lucha por la producción y la reproducción del espacio.

\section{Una propuesta metodológica para un paradigma medioambiental.}

Una perspectiva medioambiental aborda el estudio del lugar (la ciudad, por ejemplo) y de la mentalidad del lugar (la mentalidad urbana). El reconocimiento de ambos pasa por:

a) Estudiar las condiciones físicas del lugar.

b) Estudiar la participación ciudadana en la construcción material y simbólica de su espacio.

c) Averiguar cómo lo usan sus habitantes.

d) Desentrañar las estructuras de sentido y las ideologías que sustentan las formas de vida cotidiana en ese lugar.

e) Reconocer cómo los habitantes o moradores perciben, se imaginan y sueñan el entorno en el que viven.

Reconocer las condiciones materiales que mueven y promueven una determinada producción social del espacio es el aspecto más estudiado por los enfoques estructuralistas del marxismo, que ven en la competencia por el espacio uno de los aspectos sobresalientes de los conflictos sociales de la actualidad. Desde el medioambientalismo, una metodología que ha demostrado ser capaz de hacer emerger estos conflictos a través de las formas en las que los diferentes 
grupos entienden los recursos necesarios y eficientes para su supervivencia, los usos que de ellos se realizan y las luchas de intereses que la competencia por los mismos desatan, es la denominada Metodología Dambao':

- Descifra a los agentes y actores que participan en el hábitat de un lugar.

- Individualiza los recursos materiales y simbólicos y los usos de los mismos que sustentan la vida cotidiana de los actores.

- Hace emerger las consecuencias de las formas de apropiación del espacio y las problemáticas y los conflictos que de ello se derivan.

Es un método crítico que, al poner en relación actores y recursos, "pone en evidencia el escenario" en el que se desarrolla la apropiación del espacio y las consecuencias que ello tiene para el desarrollo integral del lugar y de los lugares que lo circundan. Poner límites a estos lugares desde una visión ecológica significa, al mismo tiempo, situar el marco de los problemas medioambientales a escala planetaria e identificarlos y contextualizarlos en el lugar y la cultura donde se producen.

La dimensión simbólica del espacio, que incluye la percepción, el sentido, las expectativas, las esperanzas y los sueños proyectados en él, ha ocupado un lugar un tanto marginal en los estudios socioespaciales. Adentrarse en los imaginarios sociales e individuales no es una tarea sencilla, pero sí muy fructífera para comprender el hábitat y la vida cotidiana que en él se desenvuelve. Es una clave importante para tender el puente entre las materialidades y las estructuras de sentido que lo sustentan. Lo uno sin lo otro rompe la globalidad que persigue la perspectiva medioambiental.

La inmersión en los imaginarios del habitar conduce a una lectura del lugar desgranando la relación comunicacional que espacio y sociedad entablan. El uso del lenguaje y de la escritura, los ritos convivenciales y las representaciones que estos elementos evocan, son piezas fundamentales de esta dimensión simbólica. Recorrer los caminos y vericuetos de los imaginarios ambientales conduce al investigador a situarse en la escala de quien habita.

La escala de análisis de la perspectiva medioambiental se sitúa en el grupo o en la comunidad que comparte un espacio, unas formas de apropiación del mismo y una mente local. Dentro del grupo, de las estrategias colectivas que promueve, esta perspectiva distingue el papel del individuo. Este desarrolla además su propia vivencia del lugar utilizando el espacio y proyectando en él sus expectativas. Para leer e interpretar el medio ambiente hay que situarse en el tiempo de lo cotidiano, descubriendo sus lazos con el pasado y sus proyecciones de futuro. Se trata de ver y de hacer ver los elementos comunes y universales del habitar pero descubriendo en ellos las diversidades y especificidades que diferentes culturas, comunidades e individuos construyen desde su posición social.

1. Esta metodología ha sido desarrollada para la investigación de las problemáticas medioambientales en Venezuela. 


\section{Bibliografía}

Acosta, M.; BRICEÑo LEON, R. (1987). Ciudad y Capitalismo. Universidad Central de Venezuela.

ÁNGEL, A. (1989). Hacia una sociedad ambiental. Bogotá: Editorial El Labrador.

CABANZo, F. (1993). "Alto rischio in scenari ad alta complessità territoriale: il caso di Cartagena de Indias (Colombia)" en el Convegno Scientífico La Pianificazione Ambientale dopo Rio: approcci, metodi e soggetti per i Paesi in Via di Sviluppo. Venecia, inédito.

Campiol, G. (1993). "Metodologie di pianificazione ambientale», en el Convegno Scientífico La Pianificazione Ambientale dopo Rio: approcci, metodi e soggetti per $i$ Paesi in Via di Sviluppo. Venecia, inédito.

Castells, M. (1988). La cuestión urbana. México: Siglo Veintiuno Editores.

La CeCla, F. (1993). Mente Locale. Per un'antropologia dell'abitare. Milán: Elèuthera.

LEFEBVRE, H. (1969). El derecho a la ciudad. Barcelona: Península.

Lefebvre, H. (1976). Espacio y politica. El derecho a la ciudad II. Barcelona: Península,

PARK, R.; BURGUESS, E.W.; MACKENZIE, R. (1967). La città: indicazioni per lo studio del comportamento humano nell'ambiente urbano. Milán: La città.

SAUNDERS, P. (1989). Social Theory and the Urban Question. Londres: Unwin Hyman.

SILVA, A. (1992). Imaginarios urbanos. Colombia: Tercer Mundo Editores. 